\title{
Cluster Statistical Thermodynamics (CST) - To Efficiently Calculate Quasi-Static Deformation at Finite Temperature Based on Molecular Potential
}

\author{
Ming $\mathrm{Hu}^{1,2, *}$, Haiying Wang ${ }^{1}$, Mengfen $\mathrm{Xia}^{1,3}$, Fujiu $\mathrm{Ke}^{1,4}$ and Yilong Bai ${ }^{1}$ \\ ${ }^{1}$ State Key Laboratory of Nonlinear Mechanics, Institute of Mechanics, Chinese Academy of \\ Sciences, Beijing 100080, China; *E-mail: huming@lnm.imech.ac.cn \\ ${ }^{2}$ Graduate School of Chinese Academy of Sciences, Beijing 100039, China \\ ${ }^{3}$ Department of Physics, Peking University, Beijing 100871, China \\ ${ }^{4}$ Department of Applied Physics, Beijing University of Aerospace and Aeronautics, \\ Beijing 100083, China
}

\begin{abstract}
For quasi-static deformations in engineering practice, molecular dynamics (MD) simulation requires computation resources that are not affordable even with ever-increasing computing power. In order to overcome this weakness, we developed a new method called cluster statistical thermodynamics (CST). By taking the advantage of statistical thermodynamics and adopting finite-element interpolation, the new approach can not only simulate quasi-static deformation but have very high computing efficiency. The new method is based on molecular potentials as MD does, but statistical thermodynamics help us greatly reduce the tedious calculation of thermal fluctuations of molecules. Therefore, the new method appears to be superior to MD in the simulations of quasi-static deformation. Especially CST works much more efficiently than MD with much less storage space and CPU time. In this paper, we illustrate the new methodology by means of some examples of two-dimensional quasi-static tensile process at $300 \mathrm{~K}$. It is found that the results obtained with CST are in good agreement with those obtained by fully atomistic simulations and CST is 600 times faster than MD. Hence, the new method seems to be a very efficient and promising approach to numerical simulations of solid deformations under quasi-static loadings and at finite temperatures, based on molecular potentials.
\end{abstract}

Key words: cluster statistical thermodynamics, quasi-static deformation, finite temperature, quasi-continuum method, molecular dynamics.

\section{Introduction}

Macroscopic theories of solid materials are based on statistical average of microscopic interactions and then are insufficient to deal with a system at micro- and nanoscales. In principle, mechanical behaviors of solid materials are determined by their microscopic interactions between molecules and atoms constituting them. So far,

Yilong Bai et al. (eds), IUTAM Symposium on Mechanical Behavior and Micro-Mechanics of Nanostructured Materials, 163-170.

(c) 2007 Springer. Printed in the Netherlands. 
molecular dynamics (MD) simulations, based on the full description of interaction potential of all the molecules or atoms and the Newton equation, seem to be a basic numerical approach to model these behaviors. However, despite ever-increasing computing power, we still encounter two formidable difficulties in these simulations, namely the huge gap in the length and time scales involved [1]. It is well known that even modern computers can, at most, deal with problems up to the spatial and temporal range of micrometers and nanoseconds respectively. Obviously, this can not meet the need to simulate most practical deformation processes, i.e. quasi-static deformations of samples larger than several micrometers at finite temperature. Obviously these two obstacles present a great challenge to current simulations.

During the past decade, in order to solve the length scale problem, many researchers have developed a variety of multiscale methods by imbuing continuumbased models with more-realistic atomistic details. One of the pioneering works in this field is the so-called FEAt method [2], in which the system is partitioned into a "core" (inhomogeneous) described atomistically and the "remainder" (homogeneous) treated as a continuum. Recently, Abraham et al. [3] and Broughton et al. [4] extended the method to include an additional core, handled by the quantummechanical tight-binding (TB) approximation. Although this technique has, to some extent, successfully solved the length scale problem, it can only treat dynamical process up to 20 ps so far. Therefore, it is difficult to simulate practical quasi-static processes.

Another important and remarkable approach is the quasi-continuum (QC) method developed by Tadmor et al. [5]. The QC provides a framework whereby degrees of freedom are judiciously eliminated and force/energy calculations are expedited, by means of overlaying the atomic lattice with a finite mesh (represented by repatoms) and recognizing potential energy minimization. It is particularly useful if one is primarily interested in metastable behavior of a defective material. Several researchers have successfully used this method to simulate a variety of problems, such as nanoindentation, crack tip deformation, dislocation interactions and so on. In spite of these successes, at present, the primary disadvantage of the QC method is that it is restricted to processes at absolute zero temperature $(T=0 \mathrm{~K})$. Although Shenoy and Phillips made an attempt to extend the QC method to finite temperature [6, 7], its implementation is not efficient and simulations of large problems, other than simple edge dislocations, require a much larger computational effort [7]. They performed one-dimensional Monte Carlo simulations only and noted that the obtained results raise more questions than they answer.

In this paper, we report a different and very efficient approach named cluster statistical thermodynamics (CST), to calculate quasi-static deformation of a system at finite temperature ( $T$ ) based on molecular potential. In the CST method, a system consisting of atoms is partitioned into a number of clusters determined by their nodes like finite element method. Each cluster is treated by statistical thermodynamics as an equilibrium sub-system consisting of finite atoms or molecules. In particular, we use statistical thermodynamics to greatly reduce the treatment of thermal fluctuations of molecules, which are very time-consuming in MD simulations but not closely relevant to quasi-static deformation. Based on the positions of the nodes, the Helmholtz 


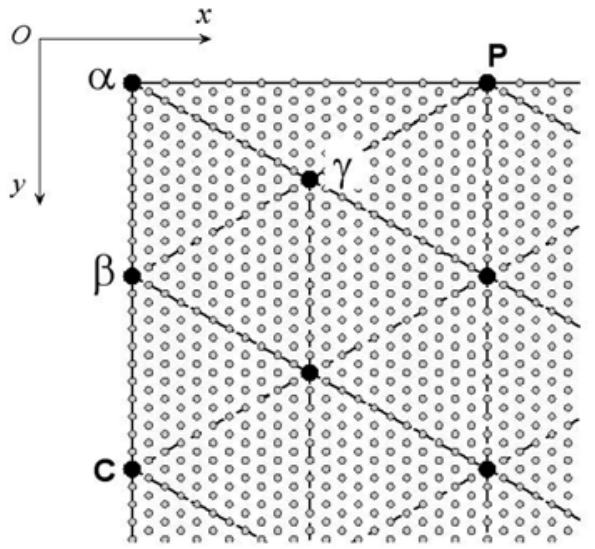

Fig. 1. Schematic of cluster construction. The small gray circles are atoms of the system and the big black circles, like $\alpha, \beta$, and $\gamma$, are the nodes which construct the clusters.

free energy of each cluster is calculated and the total free energy of the system is obtained by the summation of them. Then, the equilibrium configuration of the system can be obtained by minimizing the total Helmholtz free energy of the system with respect to the positions of nodes under the condition of constant volume or area (in three or two dimensions respectively). We assess the validity of CST by comparing a quasi-static uniaxial tension at room temperature with two fully atomistic models, i.e. molecular dynamics (MD) and "molecular statistical thermodynamics" (MST), the latter is the limit of CST with single molecule clusters.

\section{Method Formulation}

Now, we use a two-dimensional case to illustrate the principle and application of the new method. Consider a system of $N$ atoms, whose positions are denoted by $\{\mathbf{x}\}$. As shown in Figure 1, the system is partitioned into a number of clusters constructed by their corresponding nodes (like $\alpha, \beta, \gamma$, etc., see the large black circles in Figure 1).

Thus, the entire system can be characterized by all the nodes whose positions are denoted by $\{\mathbf{X}\}$. If we denote $N_{\text {node }}$ as the total of nodes, then, clearly, the freedom degree of the system can be significantly reduced since $N_{\text {node }} \ll N$. The requirement of the cluster construction is that the nodal system should behave as closely as possible to the fully atomistic system. A criterion to this requirement is that the statistical average of an observable $F$ (denoted by $\bar{F}$ ) calculated according to node positions $\{\mathbf{X}\}$ be equal to the time-average of the observable in the corresponding fully atomistic canonical system. Therefore, the aim of CST can be stated as

$$
\bar{F}(\{\mathbf{X}\})_{\mathrm{CST}}=\bar{F}(\{\mathbf{x}\})=\langle F(\{\mathbf{x}\})\rangle,
$$

where $\bar{F}$ and $\langle F\rangle$ are the time and ensemble average of $F$, respectively. 
When analyzing the mechanical response of a system under quasi-static loadings, we are required to calculate the Helmholtz free energy of the system. The free energy serves as a generating function for other mechanical quantities. Firstly, suppose that the total Helmholtz free energy $A$ of the whole system be the summation of the free energy of each cluster, i.e.

$$
A=\sum_{\alpha=1}^{N_{c}} A_{\alpha}
$$

where $A_{\alpha}$ is the free energy of cluster $\alpha$ and $N_{c}$ is the total of clusters.

Secondly, according to the local harmonic (LH) approximation [8], the Einstein model of phonon density of states in statistical thermodynamics gives the Helmholtz free energy of each cluster as

$$
A_{\alpha}=\Phi_{\alpha}+N_{\alpha} k T \sum_{\beta=1}^{2} \ln \frac{\hbar \omega_{h \beta}}{k T}=\Phi_{\alpha}+2 N_{\alpha} k T \ln \frac{\hbar\left|D_{\alpha h}\right|^{1 / 4}}{k T}
$$

where $\Phi_{\alpha}$ is the potential energy of cluster $\alpha, N_{\alpha}$ is the number of atoms within cluster $\alpha, \hbar$ is Planck's constant, $k$ is Boltzmann's constant, $T$ is temperature $(\mathrm{K})$, $\omega_{h \beta}$ and $D_{\alpha h}$ are the vibrational frequency and the local dynamical matrix of the atom $h$ adjacent to the center of cluster $\alpha$, respectively, and $\beta$, is either 2 or 3 , in accordance with dimensions.

Thirdly, we adopt local mean field approximation, i.e., assuming identical potential energy for atoms in each cluster. Then, the potential energy of cluster $\alpha$ can be expressed as

$$
\Phi_{\alpha}=N_{\alpha} \Phi_{\alpha h},
$$

where $\Phi_{\alpha h}$ is the potential energy of the atom $h$ adjacent to the center of cluster $\alpha$ and can be calculated in accord with deformed neighbors from the current interpolated displacements in the cluster. At the same time, the determinant of the local dynamical matrix of atom $h$ can be calculated as [9]

$$
\left|D_{\alpha h}\right|=\frac{\partial^{2} \Phi_{\alpha}}{\partial x_{h 1}^{2}} \frac{\partial^{2} \Phi_{\alpha}}{\partial x_{h 2}^{2}}-\left(\frac{\partial^{2} \Phi_{\alpha}}{\partial x_{h 1} x_{h 2}}\right)^{2},
$$

where $x_{h \eta}, \eta=1$ and 2, denote the positions of atom $h$ in $x$ and $y$ directions respectively.

Finally, using Equations (2-5), we can write the total Helmholtz free energy $A$ of the whole system as a function of all the node positions $\{\mathbf{X}\}$, i.e.

$$
A=A\left(\left\{\left(X_{i}, Y_{i}\right), i=1, \ldots, N_{\text {node }}\right\}\right) .
$$

We are now in a position to obtain the equilibrium configuration $\left\{\mathbf{X}_{\mathrm{eq}}\right\}$ by minimizing $A$ with respect to the nodes positions $\{\mathbf{X}\}$, when keeping the temperature $T$ and the total area of clusters, $S=\sum_{\alpha=1}^{N_{c}} S_{\alpha}$, fixed during the minimizing search. It is important to notice that the CST method described above can be straightforwardly 
applied to the deformation at absolute zero temperature. In this case, the equilibrium configuration can be easily obtained by minimizing the total potential energy of the system, $\Phi=\sum_{\alpha=1}^{N_{c}} \Phi_{\alpha}$, with respect to the nodes positions \{\} .

Here, it should be pointed out that the CST method is different from the quasicontinuum method developed by Tadmor et al. [5]. The essence of the QC method is repatom (representing a number of atoms) statics/dynamics while the CST is based on the statistical thermodynamical equilibrium of cluster nodes. Hence CST can be readily used to deformations at finite temperature.

\section{Some Examples}

We validated the CST method by simulating two-dimensional quasi-static tensile processes at $0 \mathrm{~K}$ and $300 \mathrm{~K}$. In the present work, a single hexagonally packed lattice of $\mathrm{Cu}$ with initial dimensions $68.3 \times 16.9 \mathrm{~nm}$ was uniaxially tensioned. As shown in Figure 1, the tensile direction is horizontal $(x)$, and the free lateral boundary conditions were adopted in the vertical ( $y$ ) direction, for both CST and MD simulations. The potential employed in our calculations is the Lennard-Jones potential,

$$
e(r)=4 \varepsilon_{p}\left[\left(\frac{r_{0}}{r}\right)^{12}-\left(\frac{r_{0}}{r}\right)^{6}\right]
$$

with parameters $r_{0}=2.3276 \AA, \varepsilon_{p}=0.4912 \mathrm{eV}$ [9], and the truncated distance $r_{c}=7.8379 \AA$. The total number of nodes and clusters used in CST simulations are 189 and 336 respectively, corresponding to 20549 atoms in full MD simulations. For CST, at each loading state, all the atoms reach their equilibrium positions by minimizing the total Helmholtz free energy of the whole system with respect to the node positions using the conjugate gradient method [10]. For corresponding MD simulations, it was conducted over a period of about 30ps with a time step of 2 fs using the standard Verlet algorithm [11]. For MD simulations, the 30ps period contains two parts: the first 20 ps are used to look for thermo-equilibrium and the remainder for statistical calculation of time-average positions of atoms $\left\{\mathbf{x}_{\mathrm{eq}}\right\}$. In order to facilitate the comparison of CST and MD results, we adopt the same stress definition, i.e. stress being the derivative of Helmholtz free energy. According to the LH approximation [8], the Helmholtz free energy of each loading state for MD is then computed by the determinant method (DM) [12]

$$
A_{\mathrm{MD}}=\Phi\left(\left\{\mathbf{x}_{\mathrm{eq}}\right\}\right)+2 k T \sum_{i=1}^{N} \ln \left(\frac{\hbar\left|D_{i}\right|^{1 / 4}}{k T}\right)
$$

where $\left|D_{i}\right|=\left[\omega_{i 1} \omega_{i 2}\right]^{2}$ is the determinant of the local dynamical matrix of atom $i$ and can be obtained by

$$
\left|D_{i}\right|=\left(\frac{\partial^{2} \Phi}{\partial x_{i 1}^{2}}\right)_{\mathrm{eq}}\left(\frac{\partial^{2} \Phi}{\partial x_{i 2}^{2}}\right)_{\mathrm{eq}}-\left(\frac{\partial^{2} \Phi}{\partial x_{i 1} \partial x_{i 2}}\right)_{\mathrm{eq}}^{2}
$$




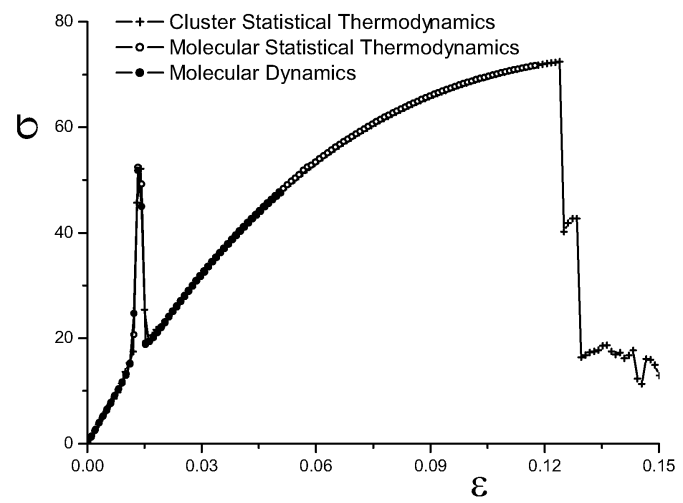

(a)

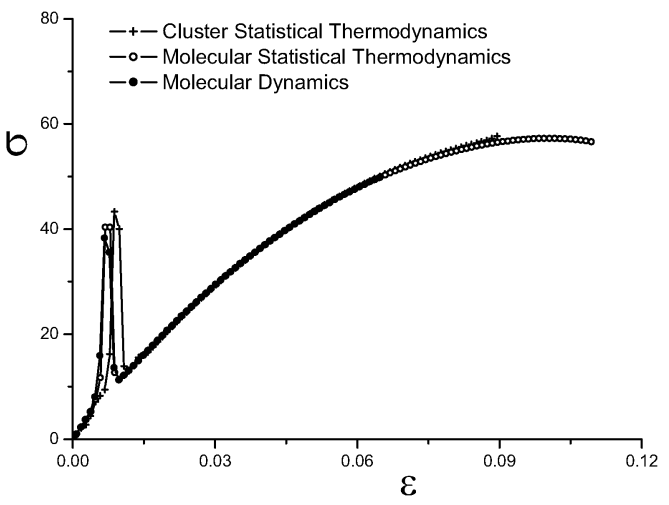

(b)

Fig. 2. (a) Stress vs strain curves at absolute zero temperature obtained by CST (+), MST (o), and $\mathrm{MD}(\bullet)$ respectively. The stress $\sigma$ is calculated by $\sigma=\left(1 / L_{y 0}\right)\left(\partial \Phi / \partial L_{x}\right)$, where $L_{x}$ and $L_{y 0}$ are the deformed length in the $X$ direction and the initial length in the $Y$ direction respectively. The unit of stress is $k \Theta / r_{0}^{2}$, where $\Theta=343 \mathrm{~K}$ is the Debye temperature for $\mathrm{Cu}$. (b) Stress vs strain curves at $300 \mathrm{~K}$. The stress $\sigma$ is calculated by $\sigma=\left(1 / L_{y 0}\right)\left(\partial A / \partial L_{x}\right)$.

Now, let us start with the comparison of the deformations at absolute zero temperature simulated by CST, MST, and MD respectively. Three stress-strain curves were calculated by differentiating the total potential energy of the system with respect to the deformed length in the $x$ direction, shown in Figure 2a. As one may expect the agreement must be excellent, since the principles of CST, MST and MD become identical at absolute zero temperature. More importantly, Figure $2 \mathrm{~b}$ shows the comparison of the three stress-strain curves at finite temperature $(300 \mathrm{~K})$ obtained by CST, MST, and MD respectively.

Figures $2 \mathrm{a}$ and $2 \mathrm{~b}$ clearly demonstrate that, before breaking, the mechanical behavior predicted by the proposed CST method almost coincides with those obtained 
by two fully atomistic simulations (MST and MD) for both absolute zero temperature and finite temperature. This indicates that the proposed CST method can accurately simulate deformation at finite temperature. In particular, the deformation simulated by CST is a quasi-static process, which corresponds to most practical tests in laboratories. The other advantage of CST simulations is that they are very efficient. For example, for all simulations performed on a PC with CPU Pentium $42.6 \mathrm{GHz}$, the calculations for each loading state took less than $10 \mathrm{~s}$ for CST and about 20 minutes for MST but 100 minutes for MD, thus CST is 600 times faster than MD simulations. Therefore, the new method seems to be a very efficient and promising approach to numerical simulation of solid deformations under quasi-static loadings and at finite temperature, based on molecular potentials.

\section{Summary}

In summary, we report in this paper a novel approach, i.e. cluster statistical thermodynamics (CST) method, to simulate deformations of crystalline solids under quasistatic loadings at finite temperature. The good agreements of uniaxial tensions obtained by CST with fully atomistic simulations validate the new method. Moreover, CST demonstrates much higher efficiency in computing time (about 3 orders less than MD). Therefore, CST appears to be a more realistic (for quasi-static loading at finite temperature) and more efficient method in simulations of solid deformation based on molecular potential.

\section{Acknowledgements}

This work is supported by the National Natural Science Foundation of China (10432050), the Chinese Academy of Sciences (KJCX2-SW-L2) and the Major State Research Project of China (G2000077305).

\section{References}

1. Kadau, K., Germann, T.C., Hadjiconstantinou, N.G., Lomdahl, P.S., Dimonte, G., Holian, B.L. and Alder, B.J. (2004) Nanohydrodynamics simulations: An atomistic view of the Rayleigh-Taylor instability, Proc. Natl. Acad. Sci. USA 101, 5851-5855.

2. Kohlhoff, S., Gumbsch, P. and Fischmeister, H.F. (1991) Crack-propagation in bcc crystals studied with a combined finite-element and atomistic model, Philos. Mag. A 64, 851878.

3. Abraham, F.F., Broughton, J.Q., Bernstein, N. and Kaxiras, E. (1998) Spanning the length scales in dynamic simulation, Comput. Phys. 12, 538-546.

4. Broughton, J.Q., Abraham, F.F., Bernstein, N. and Kaxiras, E. (1999) Concurrent coupling of length scales: Methodology and application, Phys. Rev. B 60, 2391-2403.

5. Tadmor, E.B., Ortiz, M. and Phillips, R. (1996) Quasicontinuum analysis of defects in solids, Philos. Mag. A 73, 1529-1563. 
6. Shenoy, V., Shenoy, V. and Phillips, R. (1999) Finite temperature quasicontinuum methods, Mat. Res. Soc. Symp. Proc. 538, 465-471.

7. Shenoy, V. (1998) Quasicontinuum models of atomic-scale mechanics, $\mathrm{PhD}$ thesis, Brown University.

8. LeSar, R., Najafabadi, R. and Srolovitz, D.J. (1989) Finite-temperature defect properties from free-energy minimization, Phys. Rev. Lett. 63, 624-627.

9. Najafabadi, R. and Srolovitz, D.J. (1995) Evaluation of the accuracy of the free-energyminimization method, Phys. Rev. B 52, 9229-9241.

10. Press, W.H., Teukolsky, S.A., Vetterling, W.T. and Flannery, B.P. (1992) Numerical Recipes in Fortran 77: The Art of Scientific Computing, Cambridge University Press, Cambridge.

11. Allen, M.P. and Tildesley D.J. (1987) Computer Simulation of Liquids, Oxford University Press, Oxford.

12. Hoover, W.G., Hindmarsh, A.C. and Holian, B.L. (1972) Number dependence of smallcrystal thermodynamic properties, J. Chem. Phys. 57, 1980-1985. 